\title{
La emigración cualificada española en Francia y Alemania ${ }^{1}$
}

\author{
Antonio Alaminos Chica \\ Oscar Santacreu Fernández \\ Universidad de Alicante. Instituto Universitario de Desarrollo Social y Paz \\ alaminos@ua.es \\ oscar.santacreu@ua.es
}

Recibido: 10-06-2008

Aceptado: 11-11-2008

\section{Resumen}

A pesar del interés que despierta el fenómeno de las migraciones cualificadas, es difícil encontrar datos que permitan realizar un análisis detallado de los factores que condicionan la movilidad. El caso español constituye un ejemplo de la modificación cualitativa en la composición de las migraciones de sur a norte en Europa tras el ingreso en la Comunidad Económica Europea. No se produjeron migraciones masivas de trabajadores sin cualificación; sin embargo, sí se alteró drásticamente su composición: la migración no cualificada fue sustituida por migración cualificada de España a Alemania y Francia. Esta migración queda ocultada por la llegada masiva de inmigrantes a España. Empleando los datos de la encuesta EIMSS, se analizan las diferencias de las condiciones de egreso y movilidad entre los dos tipos de migrantes, cualificados y no cualificados.

Palabras clave: migraciones cualificadas, migraciones europeas, EIMSS, emigrantes españoles.

\section{Abstract. Spanish skilled migration in France and Germany}

Despite the interest in the phenomenon of migration, it is difficult to find qualified data which allows for a detailed analysis of the factors upon which this mobility is conditioned. The Spanish case shows an important example of qualitative modification in the composition of migrations from the south to the north of Europe since Spain's introduction into the European Community. No mass migration of low skilled workers was experienced. Nonetheless, the composition underwent drastic changes: The migration of low skilled workers was replaced by that of highly skilled workers from Spain to Germany and France. This migration went unnoticed due to the mass arrival of immigrants in Spain. An analysis was made of the differences in the conditions of migration and mobility between the two types of migrants, highly and low skilled, using the data obtained in the EIMSS survey.

Key words: high skilled migrations, European migration, EIMSS, Spanish emigrants.

1. El estudio se ha realizado en el contexto de la Red de Excelencia RAMSES 2 «Réseau euro méditerranéen des centres de recherche en sciences humaines sur l'aire méditerranéenne», financiada por la Comisión Europea (VI Programa Marco de la UE). 


\section{Sumario \\ Introducción Conclusión \\ El tránsito a las migraciones cualificadas: Bibliografía}

el caso de España

\section{Introducción}

Uno de los aspectos centrales en el ámbito de las migraciones intraeuropeas, desde la década de 1990, ha sido el incremento en el número de migrantes cualificados. Así, los migrantes con nivel educativo elevado supusieron el 30\% de todas las migraciones de individuos adultos a partir de 1990, y para el año 2000 ya suponían el 35\% del stock mundial (Lowell, 2007). Este tipo de migración ha recibido diferentes nombres y tratamientos a lo largo del tiempo (Sabour, 1997), ya sea desde el punto de vista de la pérdida de capital humano de los países emisores, en la tradicionalmente llamada «fuga de cerebros» (Glaser, 1978), o evaluando la relación de este tipo de migración con el desarrollo económico de los países de origen y destino (Solé, 2001; Lucas, 2005). En los últimos años, se han desarrollado varias investigaciones sobre el tema, a partir de organizaciones como la OCDE, la ILO y LA UNCTAD. Lo que parece evidente es que la internacionalización de la economía y las TCI han acelerado el proceso de movilidad de las migraciones cualificadas. Esta asociación entre migración cualificada y desarrollo se aprecia en el desarrollo de políticas migratorias en los países desarrollados que las facilitan. Así, en el ámbito de la Unión Europea, se ha considerado la posibilidad de un sistema de "carta verde» para ciertos migrantes altamente cualificados (European Comission, 2005).

Si tenemos en cuenta la producción de investigaciones, artículos académicos y declaraciones oficiales, podría suponerse que el concepto de «migrante cualificado" (highly skilled migrant) se encuentra bien definido. Sin embargo, este concepto conserva aún una plasticidad operativa excesiva. La Comisión Global para las Migraciones Internacionales observa que «la distinción tradicional entre trabajadores cualificados y no cualificados es inútil en ciertos aspectos, y no hace justicia a la complejidad de las migraciones internacionales. Por ejemplo, en la actualidad muchos países desean contratar a los migrantes que son especialistas en tecnologías de la información e ingeniería, pero desean igualmente atraer a los migrantes que son capaces de proporcionar una atención de alta calidad a niños y personas mayores. Aunque pueden tener diferente nivel educativo, todos ellos podrían ser legítimamente descritos como trabajadores esenciales» (Global Commission for International Migration, 2005: 7).

La mayor parte de las veces, se utiliza el nivel educativo como sustituto de "cualificado», a fin de operativizar el concepto de "cualificado» con fines estadísticos. Así, Liebig y Sousa-Poza han definido "cualificado» como igual a 1 si el encuestado tiene un título universitario o un título de formación profesional y 0 en caso contrario (Liebig y Sousa-Poza, 2005). Más específicamente, Sabour considera como «cualificada» a una persona que tiene un nivel educa- 
tivo mínimo de licenciatura en un determinado campo científico e intelectual, con varios años de experiencia laboral (Sabour, 1997). Con todo, incluso tomando la educación como sustituto de "cualificado» en las poblaciones migrantes, continúan existiendo importantes deficiencias y lagunas en los datos estadísticos disponibles. Esto hace difícil comprender las complejas pautas de movilidad internacional de estos trabajadores cualificados (International Centre for Migration Policy Development, 2005).

Los años ochenta fueron testigos del comienzo de una nueva era en la política internacional, especialmente en Europa Oriental (Sabour, 1997). La caída del muro de Berlín provocó un gran éxodo de personas con alta cualificación que migraron a países como Francia, Alemania, Inglaterra y otros países desarrollados. La expectativa más probable para la mayoría de los especialistas era de grandes migraciones intraeuropeas (Bauer y Zimmermann, 1999). Previamente, se habían producido unas circunstancias muy parecidas en varios países del sur de Europa, cuando accedieron a la Unión Europea Grecia (1981), España (1986) y Portugal (1986). La adhesión de estos países ya vino entonces acompañada por las predicciones de oleadas masivas de migrantes laborales desde los países del sur de Europa hacia los demás países miembros de la Comunidad Europea. Las consecuencias que preveían los más agoreros eran que la eliminación de las barreras a la libre circulación de mano de obra, debido a las diferencias de ingresos entre estos nuevos estados miembros y la mayoría de la CE, haría bajar los salarios y se produciría un aumento del desempleo de los trabajadores nativos, al tiempo que los sistemas domésticos de seguridad social soportarían una enorme presión (Mansoor y Quillin, 2007). Pero sucedió lo contrario: los países europeos del sur pasaron, en general, de ser países de emigración neta a ser países de inmigración. En efecto, países que históricamente han sido de emigración, como España, se han convertido recientemente en países de inmigración. Hemos de considerar, en todo caso, que el concepto de migración es amplio y comprende muchos tipos diferentes de flujos migratorios y de migrantes.

La incorporación a la Comunidad Europea fue, en todo caso, decisiva para potenciar la emigración de trabajadores cualificados desde el sur hasta el centro y el norte de Europa. Se aprecian modificaciones cualitativas en las pautas de migración (Bauer, Dietz, Zimmermann y Zwintz, 2004), y las características de los flujos de migración se han hecho también más diversas: las migraciones temporales de trabajadores, especialmente de los más cualificados, están creciendo progresivamente en importancia, mientras que las redes de migración tradicional parecen perder su significación. Considerando su diversidad, la emigración de los países del sur de Europa, como España, básicamente migraciones cualificadas, queda oculta por la magnitud de la inmigración extraeuropea. Sin embargo, esta movilidad intraeuropea de baja visibilidad de trabajadores cualificados supone una realidad de cohesión social y económica de gran importancia.

Los datos más recientes no hacen sino confirmar esta realidad. Lowell analiza la distribución de migrantes adultos con educación terciaria moviéndose de 
una región a otra del mundo. Muestra un notable aumento en la concentración del movimiento de migrantes con educación terciaria en Europa a lo largo de la década. El cambio, sin embargo, es atribuido por este autor principalmente a una mayor circulación de trabajadores altamente cualificados de origen europeo que se mueven hacia otros destinos europeos (Lowell, 2007). Se trata de un fenómeno que requiere una mayor explicación. El autor considera que este incremento de la movilidad de trabajadores cualificados en Europa responde a dos procesos: primero, que Europa se hizo más atractiva para trabajadores cualificados que antes migraban a otras zonas, como América del Norte; segundo, que, durante la última década, la movilidad intraeuropea puede haber aumentado como consecuencia de un fortalecimiento de la integración en la Unión Europea.

\section{El tránsito a las migraciones cualificadas: el caso de España}

En los últimos años, España se ha convertido en un país que recibe emigración en lugar de generarla, tal y como ha sido frecuentemente mencionado. Este cambio de tendencia se enmarca dentro de un proceso de declive en la emigración española. Como muestra de este declive, a finales del año 2007, el número de ciudadanos españoles residentes en Francia y Alemania era, respectivamente, de 159.825 y de 83.134 (Ministerio de Trabajo y Asuntos Sociales, 2007). En general, las tasas de emigración en los países del sur de Europa comenzaron a descender a principios de los años setenta, y nunca han vuelto a recuperar la dinámica anterior, ni siquiera tras la entrada de estos países en la Unión Europea; sin embargo, la composición de la migración ha experimentado un cambio sustantivo, como puede apreciarse en los datos procedentes de la encuesta EIMSS (European Internal Mobility Social Survey), construida en el contexto del proyecto de investigación PIONEUR ${ }^{2}$ del V Programa Marco de la Unión Europea. En el gráfico siguiente (figura 1), los datos expresan el porcentaje de cada tipo de emigrante para cada año.

La tendencia evidente es un incremento lineal en la migración de españoles cualificados, que sustituye a la emigración de trabajadores no cualificados. La posible estacionalidad que muestra la serie antes de la incorporación a la Comunidad Europea (entre 1974 y 1986), se rompe claramente. En base a la serie temporal, puede concluirse, pues, que la tendencia de crecimiento en la migración cualificada de españoles aumenta tras la incorporación a la CE. Dos preguntas importantes que nos podemos plantear son las siguientes: ¿cuáles son, al margen del nivel de cualificación, las diferencias entre estos dos colectivos? ¿Se plantean ambos colectivos el fenómeno de la movilidad dentro de parámetros semejantes o diferentes?

2. The PIONEUR Project: Pioneers of Europe's Integration "from Below»: Mobility and the Emergence of European Identity among National and Foreign Citizens in the EU, European Comission, Fifth Framework Programme, Key Action «Improving the Socio-economic Knowledge Base», página web: <obets.ua.es/pioneur>. 


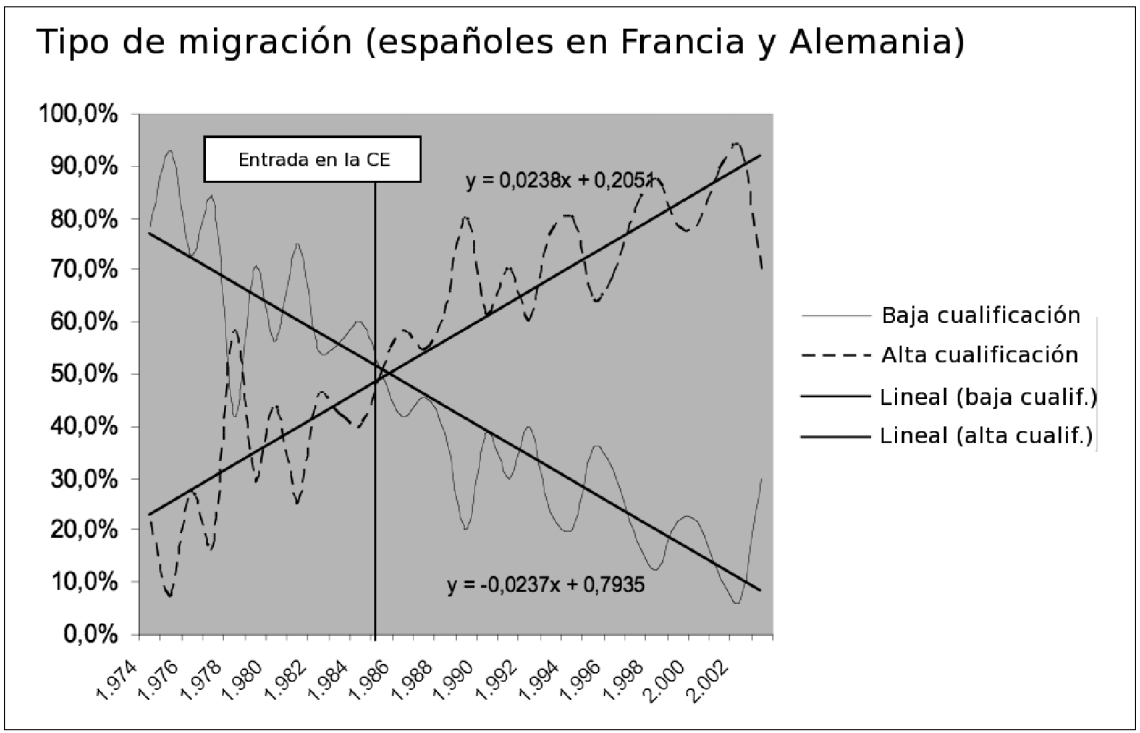

Figura 1. Porcentajes de migración cualificada y no cualificada de españoles en Francia y Alemania. Fuente: EIMSS.

El modelo explicativo que proponemos intenta analizar las diferencias que, en cuanto a actitudes y comportamientos, existen entre los migrantes españoles cualificados y los no cualificados que residen en Alemania y Francia, en particular, la existencia de una diferencia importante en la disposición a la movilidad.

Nuestra hipótesis principal consiste en que el grado de cualificación del migrante es un factor explicativo determinante, respecto, por un lado, a su disposición hacia la movilidad, y, por otro, a la expectativa de un futuro retorno a su país de origen. Consideramos, además, que el estatus de los padres explica esta mayor movilidad de los migrantes cualificados. Uno de los elementos importantes en este planteamiento es el estatus familiar: los migrantes cualificados proceden, con mayor probabilidad, de familias cualificadas que han facilitado las experiencias de movilidad previas. En ese sentido, una de las diferencias evidentes es que, en el caso de los migrantes cualificados, la movilidad ha perdido su carácter dramático y cuenta con un grado de apoyo familiar muy superior al referido por los migrantes no cualificados, dado que los primeros consideran la migración como positiva. Concretamente, el 50\% de las familias de los migrantes cualificados considera muy positiva dicha experiencia, frente al $29 \%$ de las familias de los no cualificados. En general, los migrantes cualificados muestran una mayor competencia intercultural, expresada en términos de competencia lingüística, así como una mayor flexibilidad frente a la movilidad. Por el contrario, los trabajadores no cualificados muestran una menor predisposición a la movilidad. 


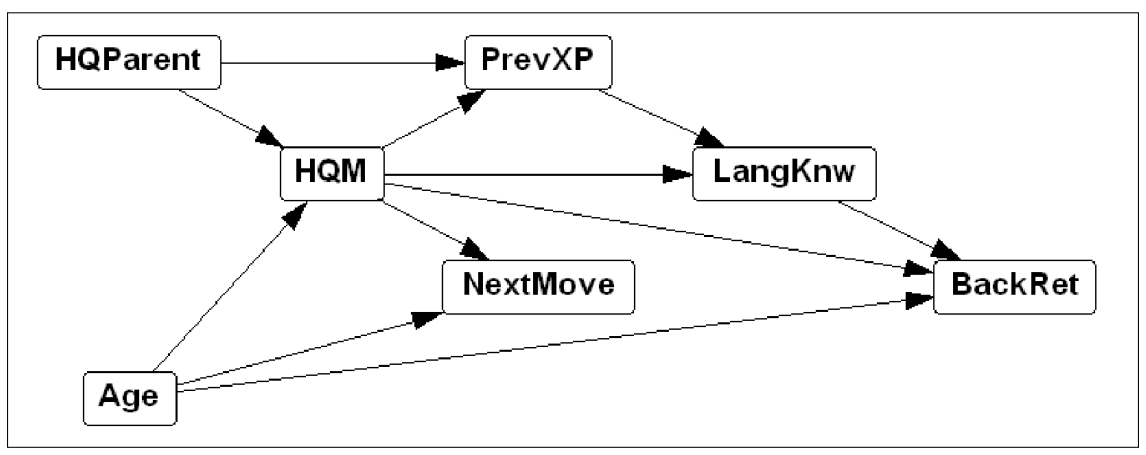

Figura 2. Diagrama causal del modelo teórico sobre migraciones cualificadas. Fuente: elaboración propia.

A partir de los datos de la EIMSS, hemos seleccionado una serie de variables en función de su capacidad explicativa y de su relación con las migraciones cualificadas. El diagrama del modelo teórico que proponemos es el siguiente (figura 2).

Como variables exógenas, consideramos la edad del encuestado (Age) y la cualificación de sus padres (QHParent). Ésta última es una variable dicotómica que refleja el hecho de que el padre del encuestado, o la madre, o ambos, tengan estudios superiores (universitarios). Por otro lado, los datos no nos permiten establecer una relación estadísticamente significativa de la variable género con el resto de variables estudiadas, por lo que dicha variable no ha sido considerada para ser incluida en el modelo. La variable central del modelo es la que se refiere al hecho de que el encuestado esté o no en el grupo de las migraciones cualificadas $(H Q M)$. Siguiendo la definición de migraciones cualificadas expuesta anteriormente, esta variable dicotómica ha sido construida de modo que un encuestado forma parte de este grupo cuando tiene estudios universitarios. Ciertamente, el nivel educativo puede considerarse una variable relevante en el ámbito de la movilidad europea. Por otro lado, es innegable que, para los migrantes cualificados, el reconocimiento de diplomas y cualificaciones en todos los estados miembros de la UE constituye una condición de gran importancia. En este sentido, el artículo 47 (ex 57) del Tratado de la CE establece la necesidad del mutuo reconocimiento de diplomas, certificados y otras evidencias de cualificación formal, a fin de facilitar a las personas el acceso y el desempeño de actividades como personas autoempleadas. Desde un punto de vista práctico, los mecanismos para el reconocimiento de los diplomas fueron implementados de forma gradual, con lo cual se creó un nuevo método de reconocimiento profesional.

Otra variable considerada en el modelo es la existencia o no de experiencias previas de movilidad del encuestado en terceros países (PrevXP), esto es, cualquier país distinto al de origen y al de residencia actual. En efecto, las personas 
cualificadas tienen un alto nivel de movilidad en Europa, en comparación con las personas con cualificación baja o media en la UE en general (Krieger y Fernandez, 2006). Los datos de la EIMSS corroboran esta afirmación.

También hemos considerado como indicador de competencia intercultural el conocimiento previo del idioma del país en el que reside actualmente el encuestado (LangKnw) y, finalmente, hemos incluido en el modelo dos variables que, en cierto modo, recogen las intenciones de movilidad futura del encuestado: la primera de ellas es su disposición a migrar a un tercer país, si con ello mejora su nivel de vida (NextMove); la segunda variable recoge la expectativa de retorno al país de origen cuando el encuestado llegue a la edad de la jubilación laboral (BackRet).

Para determinar en qué grado los datos sustentan las hipótesis de partida, utilizamos los modelos estructurales de covarianzas. Esta técnica estadística multivariante se utiliza habitualmente para estudiar y analizar las relaciones de dependencia, en términos de carga explicativa, que existen entre las variables que forman parte de un fenómeno social determinado. Este método de análisis define un modelo como un conjunto de ecuaciones estructurales que podemos representar mediante un diagrama causal. Nuestra hipótesis, expresada como ecuación, establece una relación explicativa entre las variables exógenas «edad» $\mathrm{y}$ «educación superior de los padres» con la cualificación del migrante:

$$
\begin{aligned}
& \mathrm{HQM}=-0,012^{*} \text { Age }+0,42^{*} \mathrm{HQParent} \text {, Errorvar. }=0,17, \mathrm{R}^{2}=0,28 \\
& (0,0020) \quad(0,046) \quad(0,012) \\
& \begin{array}{lll}
-5,89 & 9,18 & 14,47
\end{array}
\end{aligned}
$$

Donde, como hemos dicho anteriormente, $H Q M$ es la variable dicotómica que identifica a los migrantes cualificados, Age es la edad del encuestado y HQParent una variable dicotómica referida a la formación de los padres con los valores posibles 1 (los padres del encuestado tienen formación superior) y 0 (los padres no tienen formación superior). Podemos apreciar cómo las pruebas $t$ para cada coeficiente nos indican que ambas relaciones propuestas son significativas $(-5,89$ y 9,18 , respectivamente), así como una varianza total explicada de un $28 \%$. Se observa también que la variable que recoge el nivel formativo de los padres muestra una importante carga a la hora de explicar la cualificación del encuestado $(0,42)$. Evidentemente, a mayor formación de los padres, más probable es que nos encontremos ante un migrante cualificado. Como apreciábamos anteriormente, la educación de los padres influye en el modo en que se vive familiarmente la emigración: el mayor grado de formación de los padres, y su previsible mayor cosmopolitismo, hacen que se considere la emigración en términos menos dramáticos. La educación de los hijos en segundas y terceras lenguas es también un factor decisivo. De este modo, la posible movilidad de los cualificados se ve incentivada, o al menos no frenada, en el entorno familiar. La relación entre la edad del entrevistado y su cualificación viene claramente a expresar el cambio temporal en el tipo de migrante. Los migrantes sin cualificación tienen en la actualidad más edad que los más recientes (los cualificados). En ese sentido, la edad expresa indirectamente el momen- 
to de la migración, en la medida que las edades de los entrevistados en el momento de la migración son muy parecidas.

En la comparación que estamos estableciendo entre los dos tipos de migrante, podemos apreciar la relación significativa que existe entre la diferente cualificación y las condiciones de llegada al país de destino, así como la disposición a la movilidad. Dentro del modelo, la variable PrevXP, que recoge la existencia de experiencias previas de movilidad en otros países, expresa los diferentes perfiles de emigración. Los migrantes cualificados, $H Q M$, con un coeficiente de $0,21(t 4,27)$, muestran una movilidad previa superior a los no cualificados. Esta experiencia previa está asociada tanto a programas formativos como al apoyo familiar. La dependencia de la formación de los padres se aprecia en la relación significativa entre el nivel educativo de los padres y el de los migrantes. En conjunto, los migrantes cualificados muestran una experiencia de movilidad muy superior a la de los no cualificados, en gran parte explicada por el estatus educacional de los padres. Esta afirmación referida al pasado es consistente con las expectativas y la disposición a la movilidad futura, como podremos apreciar a continuación:

$$
\begin{array}{ccc}
\text { PrevXP }= & 0,21^{*} \mathrm{HQM}+ & 0,15 * \mathrm{HQParent}, \text { Errorvar. } \\
(0,049) & (0,052) & 0,19, \mathrm{R}^{2}=0,10 \\
4,27 & 2,86 & (0,013) \\
& & 14,47
\end{array}
$$

Otro rasgo diferencial entre los cualificados y los no cualificados es el de poseer una mayor competencia intercultural. El conocimiento previo del idioma del país de residencia actual es significativamente más elevado entre los migrantes cualificados. Esto supone una ventaja especial en términos de inserción socioeconómica. Esta mayor competencia intercultural se suma a la mayor experiencia previa de movilidad mencionada anteriormente. Los migrantes cualificados son, en ese sentido, un grupo con elementos mucho más cosmopolitas que los no cualificados. La correspondiente ecuación estructural nos muestra que ambas relaciones son estadísticamente significativas en los encuestados ( $t$-value 3,39 y 3,23, respectivamente), con una carga similar $(0,15)$. Veámoslo:

LangKnw $=0,15^{*} \mathrm{HQM}+0,15^{*} \operatorname{PrevXP}$, Errorvar. $=0,17, \mathrm{R}^{2}=0,069$
$(0,043)$
$(0,046)$
3,39
3,23
$(0,012)$
14,47

La expectativa de volver tras la jubilación al país de origen aparece en el $76,8 \%$ de los encuestados. Sin embargo, el ajuste sobre los datos de la EIMSS nos muestra que esta expectativa es significativamente menor para los migrantes cualificados $(-0,18, t=-4,04)$, así como para aquéllos con una mayor competencia intercultural $(-0,097, t=-2,06)$. Evidentemente, la mayor competencia intercultural de los cualificados sugiere una mayor facilidad de integración sociocultural. Esto podría implicar, a su vez, una menor expectativa de retorno. Esta expectativa se complementa con el tiempo de residencia en el país de destino, de modo que el deseo de regresar también es menor cuanto mayor es la edad de los encuestados, aunque con un menor coeficiente explicativo 
$(-0,010, t=-5,08)$. Podríamos encontrar la explicación en el factor de tiempo de residencia en el país de destino. Teniendo en cuenta que las medias de edad en el momento de la migración son bastante equivalentes, la mayor edad del emigrante supone, en general, un mayor tiempo de residencia y, por lo tanto, una previsible mayor integración social (Alaminos, 2006):

BackRet $=-0,18^{*} \mathrm{HQM}-0,097^{*}$ LangKnw $-0,010^{*}$ Age, Errorvar. $=0,16, \mathrm{R}^{2}=0,084$

$$
\begin{array}{llll}
(0,044) & (0,047) & (0,0020) & (0,011) \\
-4,04 & -2,06 & -5,08 & 14,47
\end{array}
$$

Por último, la disposición del encuestado a desplazarse a otro país, si con ello mejora su nivel de vida, recogida en la variable NextMove, es un rasgo diferencial en los migrantes cualificados (con un coeficiente de 0,10 y $t=2,21$ ). Esta disposición estaría también explicada de forma estadísticamente significativa por la edad del encuestado (a menor edad, mayor disposición, en términos de cambio medio). Ambas variables, edad y cualificación, poseen una estrecha relación. En ese sentido, la edad viene a sugerir una interacción por la cual los más jóvenes entre los migrantes cualificados muestran una mayor probabilidad de movilidad futura:

NextMove $=0,10^{*} \mathrm{HQM}-0,0083^{*}$ Age, Errorvar. $=0,18, \mathrm{R}^{2}=0,067$
$(0,046)$
$(0,0021)$
2,21
$-3,89$
14,47

En conjunto, la explicación puede expresarse mediante el siguiente diagrama (figura 3), en el que hemos incorporado los coeficientes de ajuste.

Los coeficientes de bondad del ajuste nos muestran que las diferentes hipótesis dan lugar a una explicación consistente. El chi-square, una medida de ajuste global del modelo, es bajo comparado a los grados de libertad, lo cual indica un buen ajuste. Otros indicadores que complementan la dependencia de

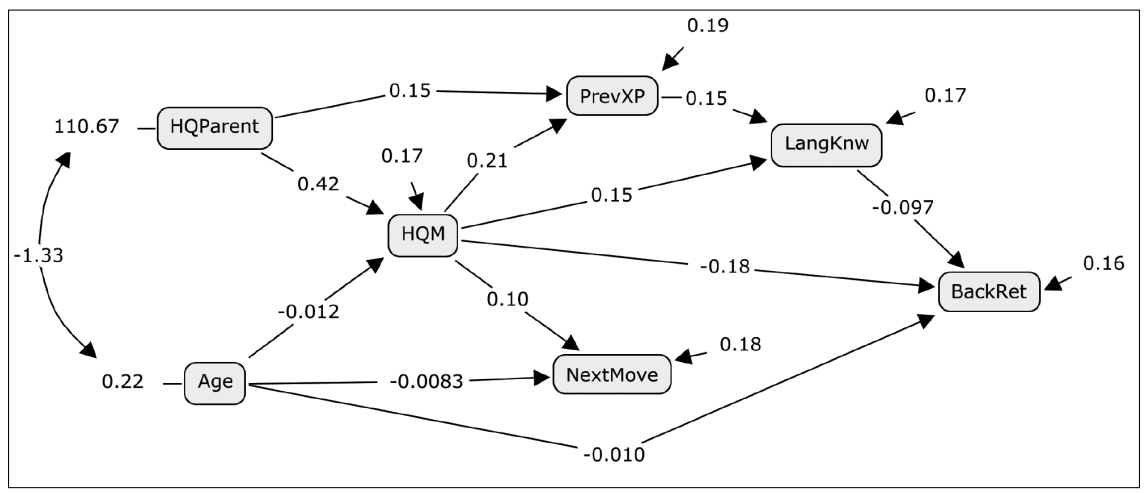

Figura 3. Diagrama causal del modelo teórico sobre migraciones cualificadas con coeficientes de ajuste. Chi-square $=7,47, \mathrm{df}=9, \mathrm{P}$-value $=0,58872$, RMSEA $=0,000$. Fuente: elaboración propia. 
chi-square del tamaño muestral expresan también un buen ajuste de nuestra explicación a partir de los datos disponibles $(0,58) .{ }^{3}$

\section{Conclusión}

El estudio de las migraciones cualificadas ha adquirido, por sus particulares características, una gran importancia en el contexto de los estudios sobre migraciones. A mediados de los años ochenta, el acceso de varios países del sur de Europa a la Comunidad Europea y la consiguiente desaparición de las barreras a la movilidad laboral no produjeron una migración masiva de sur a norte, sino que los países europeos del sur pasaron de ser países de emigración neta a ser países de inmigración neta (especialmente en el caso de España). Paralelamente, la incorporación a la CE potenció la emigración de trabajadores cualificados desde dichos países hacia el centro y el norte de Europa. Estas migraciones cualificadas dentro del ámbito de la Unión Europea suponen un importante factor de cohesión socioeconómica. En el caso español, se observa, precisamente a partir de la incorporación de dicho país a la Comunidad Europea, que la composición de la emigración experimenta un cambio sustantivo, al producirse un incremento lineal de las migraciones cualificadas de españoles que sustituye a la emigración de trabajadores no cualificados.

Para el caso de los españoles, se concluye que el grado de cualificación del migrante es un importante factor explicativo respecto a su disposición y a sus expectativas de movilidad. Específicamente, en el caso de los migrantes cualificados, la movilidad ha perdido su carácter dramático, debido, entre otros factores, a una mayor competencia intercultural, al apoyo familiar y a programas formativos. Muestran también un nivel de movilidad previa en Europa muy superior a la movilidad de los migrantes no cualificados. Esta mayor movilidad previa de los migrantes cualificados, unida a su mayor competencia intercultural en términos de conocimiento previo del idioma, suponen una ventaja especial en términos de inserción socioeconómica y cultural que se refleja en una menor expectativa de retorno al país de origen al final de la vida laboral. Consecuentemente, los migrantes cualificados muestran también, como rasgo diferencial, una mayor disposición a futuras experiencias migratorias con el objetivo de mejorar su nivel de vida.

3. P .58 y RMSEA = 0,00. Los índices GFI (Goodness of Fit Index) y AGFI (Adjusted Goodness of Fit Index) presentan unos valores de 0,99 y 0,98, respectivamente. 


\section{Bibliografía}

Alaminos, Antonio (2006). Living abroad. Intraeuropean migrations. Alicante: Pioneers of Europe. WP 14.

Alaminos, Antonio; Braun, Michael; Muxel, Anne y Tambini, Damian (coords.). The PIONEUR Project. Pioneers of Europe's Integration "from Below»: Mobility and the Emergence of European Identity among National and Foreign Citizens in the EU [en línea]. European Comission: Fifth Framework Programme, Key Action «Improving the Socio-economic Knowledge Base». Website <obets.ua.es/pioneur>.

BAUER, T. y Zimmermann, K. (1999). Assessment of possible migration pressure and labour market impact following EU enlargement to central and eastern Europe. Bonn: IZA Research Report 3.

Bauer, T.; Dietz, B.; Zimmermann, K. F. y Zwintz, E. (2004). «German migration: development, assimilation and labor market effects». En: K. F. ZIMMERMANN (ed.). European migration: What do we know? Oxford, UK: Oxford University Press.

EUROPEAN COMISSION (2005). Green paper on the management of economic migration. Glaser, W. (1978). The Brain Drain: Emigration and return. Oxford: Pergamon.

Global Commission for InTERnational Migration (2005). Migration in an interconnected world. New direction for action, octubre, p. 7.

InTERnATIOnal CENTRE For Migration POlicy Development (2005). Highly skilled migration. Fourth coordination meeting on international migration, octubre.

KRIEGER, H. y FERNÁNDEZ, E. (2006). Too much or too little long-distance mobility in Europe? EU policies to promote and restrict mobility. Foundation seminar on worker mobility. Riga, 7 de junio. Dublín: European Foundation for the Improvement of Living and Working Conditions.

Liebig, T. y SousA-PozA, A. (2005). «Taxation, ethnic ties and the location choice of highly skilled immigrants». OECD Social Employment and Migration Working Papers, 24. OECD Publishing.

LOWELL, B. (2007). «Trend in international migration flows and stocks, 1975-2005». OECD Social Employment and Migration Working Papers, 58. OECD Publishing.

LUCAS, Robert E. B. (2005). International migration regimes and economic development. Cheltelham: Edward Elgar Publishing.

Mansoor, A. y Quillin, B. (2007). Migration and remittances: Eastern Europe and the former Soviet Union January. The World Bank.

Ministerio de Trabajo y Asuntos Sociales (2007). Datos CERA a 1 de octubre de 2007. Secretaría de Estado de Inmigración y Emigración. Gobierno de España.

SABOUR, M. (1997). "The North African intellectual diaspora in the nordic countries. The socio-political factors of expatriation». En: SABOUR, M'Hammed y VIKOR, Knut. Ethnic encounter and culture change. Papers from the Third Nordic Conference on Middle Eastern Studies. Londres: Hurst \& Co Publishers.

SKELDON, Ronald (1997). Migration and development: A global perspective. AddisonWesley.

SolÉ, C. (coord.) (2001). El impacto de la inmigración en la economía y en la sociedad receptora. Barcelona: Anthropos. 